\title{
Optical-Switch-Enabled Microfluidics for Sensitive Multichannel Colorimetric Analysis
}

\author{
Jiukai Tang, Xiaobao Cao, Guangyu Qiu, Andrew deMello, and Jing Wang* \\ Cite This: Anal. Chem. 2021, 93, 6784-6791 \\ Read Online
}

ABSTRACT: The implementation of colorimetric analysis within microfluidic environments engenders significant benefits with respect to reduced sample and reagent consumption, system miniaturization, and real-time measurement of flowing samples. That said, conventional approaches to colorimetric analysis within microfluidic channels are hampered by short optical pathlengths and single-channel configurations, which lead to poor detection sensitivities and low analytical throughputs. Although the use of multiplexed light source/ photodetector modules allows for multichannel analysis, such configurations significantly increase both instrument complexity and cost. To address these issues, we present a four-channel colorimetric

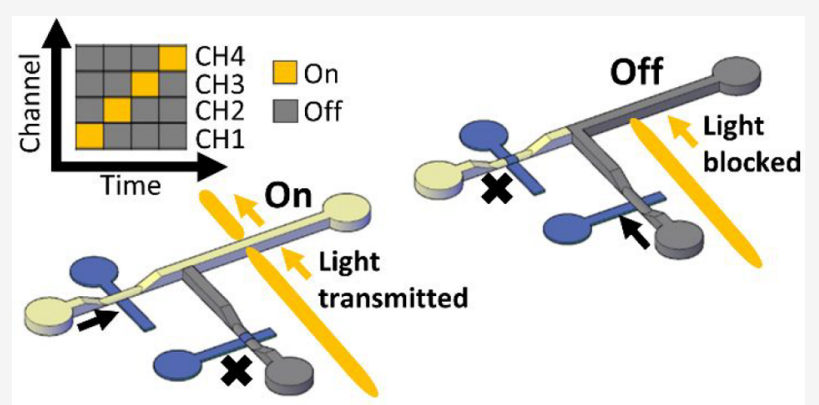
measurement scheme within an optical-switch-enabled microfluidic chip (OSEMC) fabricated by two-photon stereolithography. The integration of optical switches enables sequential signal readout from each detection channel, and thus, only a single light source and a photodetector are required for operation. Optical switches can be controlled in a bespoke manner by changing the medium in the switch channel between a "light-transmitting" fluid and a "light-blocking" fluid using pneumatic microvalves. Such optical switches are characterized by fast response times (approximately $200 \mathrm{~ms}$ ), tunable switching frequencies (between 0.1 and $1.0 \mathrm{~Hz}$ studied), and excellent stability. Operational performance demonstrates both good sensitivity and reproducibility through the colorimetric analysis of nitrite and ammonium samples using four detection channels. Furthermore, the use of OSEMC for parallel and real-time analysis of flowing samples is investigated via characterization of the adsorption kinetics of tartrazine on activated charcoal and the catalytic reaction kinetics of horseradish peroxidase (HRP).

\section{INTRODUCTION}

Colorimetric detection is a ubiquitous method for sample analysis in a wide variety of applications, such as environmental and food quality monitoring, ${ }^{1-6}$ pathogen detection, ${ }^{7,8}$ and the characterization of metabolic activities." 10 The implementation of colorimetric analysis within microfluidic environments engenders the advantages of low sample and reagent consumption, system miniaturization, and convenient measurement of flowing samples. Compared with the 96-well-platebased colorimetric analysis platforms, higher sensitivities and improved limits of detection can be realized within microfluidic systems through the use of extended detection channel lengths, ${ }^{11}$ multiple reflection schemes, ${ }^{12,13}$ or coiled optical fibers. ${ }^{14}$ That said, conventional microfluidic formats usually comprise only a single detection channel, leading to low analysis efficiencies. In this regard, the development of multichannel microfluidic devices for colorimetric analysis represents a direct route to enhancing analytical throughputs. The simplest approach in this regard involves a parallel configuration of light source/photodetector modules across multiple channels. For example, Lynch et al. ${ }^{15}$ used 13 photodiodes to develop a multichannel UV absorbance detector for throughput-enhanced two-dimensional chromatographic separations. In addition, Sieben and co-workers ${ }^{1}$ developed a four-channel microfluidic chip for nitrite quantification, where four light source/detector pairs were configured along the flow channel for monitoring both the reaction process and the efficiency of reagent mixing. Despite their obvious utility, such parallel configurations substantially increase the total cost and complexity of the device especially when sensitive light sources or spectrometers are needed. Charge-coupled device (CCD) imaging can be another approach for multichannel optical applications. For example, Zhang et al. $^{16}$ and Pan et al. ${ }^{17}$ developed a CCD-based detector to record the intensity variations of light emitted from 12 - 20 fibers for pressure sensing using a wearable device. Despite high analysis throughputs, the imaging method may cause a low resolution and cannot provide the spectrum information for colorimetric measurements.

Received: February 12, 2021

Accepted: April 7, 2021

Published: April 20, 2021 


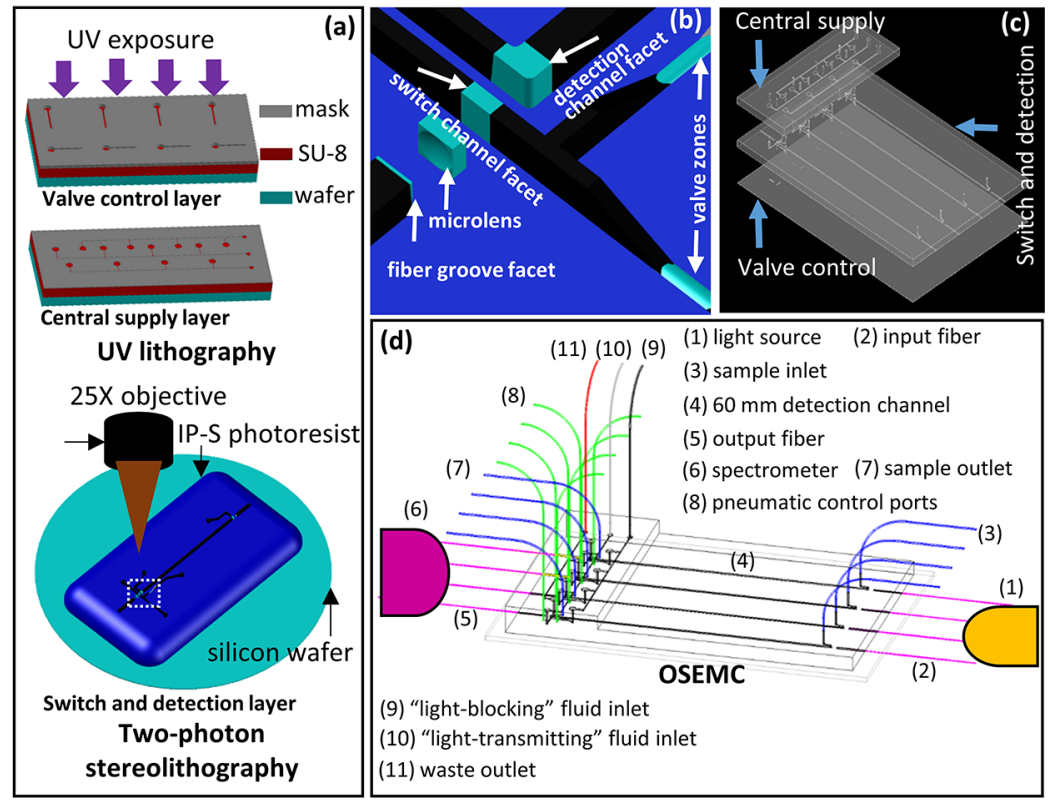

Figure 1. Fabrication and structure of the OSEMC system. (a) UV lithography was used to fabricate molds containing 2D planar structures (for the valve control layer and central supply layer), while two-photon stereolithography was used to fabricate molds of the switch and detection layers. (b) Enlargement of the region corresponding to the white dashed box in part a showing the key optical structures and microvalves (colored cyan) printed with the hierarchical printing strategy. (c) An exploded view of three assembled layers that comprise the OSEMC. (d) An overall schematic of the OSEMC system for multichannel colorimetric analysis. Detailed annotations are included in the image.

An alternative approach to achieve multichannel applications using a single light source and photodetector is by optical switches which enable each detection channel to work in sequence. Microelectromechanical systems (MEMS) and optofluidic systems are two typical technologies realizing optical switches. MEMS-based optical switches can operate with switching times of only a few milliseconds via electrostatically or magnetically controlled mirror angles. ${ }^{18}$ Despite their utility, such a system represents an additional unit and the device cost increases significantly with the number of switch ports. This makes it challenging to integrate MEMS-based switches within microfluidic systems for multichannel colorimetric analysis. In contrast, optofluidic systems have clear advantages with respect to flexibility, since the optical switch can be integrated via direct modulation of fluid (e.g., via pumping, electrowetting, and pneumatics) at no extra cost. Both light reflection and absorption by fluids can form the basis of an optofluidic switch, through the modulation of the direction or transmittance of light. The phenomenon of total internal reflection (TIR) can be used to abruptly change the direction of light through careful choice of the light incident angle and media refractive indices at a dielectric interface. Using this principle, optical switches have been realized within a range of microfluidic systems. ${ }^{19-22}$ That said, for such reflection-based switches, due to the need to sensitively control the incident angle to realize TIR, complex structure layouts must be created for multichannel applications. In contrast, for light-absorption-based optical switches, fluidic layouts can be simplified because of the independence on the incident angle of light. According to the Beer-Lambert law, at sufficiently high chromophore concentrations, the amount of light transmitted through a fluid sample can be made vanishingly small, and thus, the fluid may function as a "light-blocking" object. On the other hand, a transparent fluid will have no effect on light transmittance and acts as a "light-transmitting" object. As one application example based on light absorption, electrowetting-controlled droplet deformation can induce the change of filling medium in an optical cell to open or close the optical path. Accordingly, this system can function as an optical switch for possible applications in light shutters, attenuators, and displays. ${ }^{23-25}$ However, issues related to dielectric breakdown and complexity associated with the fabrication of electrode networks severely limit its widespread application. ${ }^{26,27}$ In addition to such an electrowetting system, "lightblocking" and "light-transmitting" fluids can be regulated using pumps. For example, Kim et al. used a pneumatic pumping system to load a strongly absorbing dye solution and deionized water to selectively block and enable light transmittance. Such a system allowed the high-throughput study of the influence of light conditions on microalgal oil production. ${ }^{28}$

The dynamic manipulation of "light-transmitting" and "lightblocking" fluids using an appropriate pumping system is critical for the practical application of optical switches and characterized by tunable switching frequencies, operational automation, and reliability. Droplets can be continuously generated in a two-phase system using syringe pumps to modulate the light. ${ }^{19}$ Nevertheless, it is relatively difficult to manipulate and position individual droplets in a bespoke manner, thus limiting the user's ability to precisely control multiple optical switches. Alternatively, droplet manipulation can be achieved through the use of pneumatic microvalves which are powerful tools for controlling fluids in microfluidic channels. ${ }^{29,30}$ Indeed, they have been used to excellent effect in a range of biochemical applications, ${ }^{31}$ including droplet combination and dilution, ${ }^{32,33}$ cell screening and sorting, ${ }^{34,35}$ and nucleic acid purification ${ }^{36}$ due to their programmable nature. Normally, the microvalve molds can be fabricated via UV lithography and thermal reflow methods. ${ }^{37,38}$ However, this approach has a limited utility in fabricating complex structures in a single step. Additionally, valve molds made via 
UV lithography and reflow normally range between 10 and 30 $\mu \mathrm{m}$ in height and have curved surfaces with the out-of-plane curvature, ${ }^{29,38}$ while, for bare-fiber optical applications, fiber groove molds having heights in excess of $125 \mu \mathrm{m}$ and planar end facets are required. ${ }^{11,39,40}$ In recent years, two-photon stereolithography has been shown to be a powerful technique for the creation of complex, three-dimensional structures on micrometer and sub-micrometer scales, ${ }^{11,41,42}$ enabling the direct fabrication of sophisticated structures of variable dimensions, geometry, and curvature.

In the current study, we make use of two-photon stereolithography to present an optical-switch-enabled microfluidic chip (OSEMC) for multichannel sensitive colorimetric analysis. After fabricating and assembling the OSEMC system, we assessed its utility in multichannel colorimetric analysis. The performance of the optical switches was characterized in terms of the response time, tunability of switching frequency, and operational stability. Subsequently, sensitivity and reproducibility between multiple detection channels were evaluated through the analysis of nitrite and ammonium samples. Finally, the OSEMC system was used for parallel and real-time analysis of flowing samples, specifically through the characterization of the adsorption kinetics of tartrazine on activated charcoal and the catalytic reaction kinetics of horseradish peroxidase (HRP).

\section{EXPERIMENTAL SECTION}

2.1. System Configuration. The OSEMC was manufactured via mold replication. Representative images are presented in Figure S1, showing molds for the control layer (Figure S1a), the central supply layer (Figure $S 1 b$ ), and the switch and detection layer (Figure S1c). The first two molds (Figure S1a and b) containing 2D planar structures were manufactured by UV lithography using an SU-8 photoresist (Figure 1a), while the third mold (Figure S1c) containing 3D structures such as microlenses and microvalves was fabricated by two-photon stereolithography (Nanoscribe, Karlsruhe, Germany) using IP$S$ photoresist (Figure 1a) and hierarchical modular printing. The key optical structures and valve structures in the third mold are colored cyan in Figure $1 \mathrm{~b}$ and were printed with the following high-resolution parameters: slicing distance $-0.2 \mu \mathrm{m}$, hatching distance $-0.2 \mu \mathrm{m}$, and laser dose $-40 \%$. Other parts of this mold were fabricated using common printing parameters: slicing distance $-1.0 \mu \mathrm{m}$, hatching distance -0.5 $\mu \mathrm{m}$, and laser dose - 70\%. A more detailed discussion of the hierarchical modular printing process can be found elsewhere. ${ }^{11}$ The printing process (the polymerization of photoresist) and key structures printed in the photoresist prior to development are shown in video S1 and Figure S2, respectively. After the fabrication of the three molds, three layers of polydimethylsiloxane (PDMS) were manufactured via standard mold replication methods. An exploded view of the assembled PDMS layers is schematically shown in Figure 1c. The entire analysis system consisted of a MNWHD2 white light LED (Thorlabs, Newton, USA), the OSEMC, FG105UCA multimode optical fibers (Thorlabs, Newton, USA), a ULS2048LTEC spectrometer (Avantes, Apeldoorn, Netherlands), a solenoid valve for pneumatic control, and a four-channel REGLO Digital MS-4/6 peristaltic pump (ColeParmer, Wertheim, Germany). Input light was guided via the four optical fibers from the common light source to four parallel detection channels in the OSEMC. Each detection channel was $60 \mathrm{~mm}$ in length, $200 \mu \mathrm{m}$ in width, and $250 \mu \mathrm{m}$ in height. Light from the detection channel passed through a switch channel ( $100 \mu \mathrm{m}$ in width and $250 \mu \mathrm{m}$ in height), which allows light to be selectively transmitted or blocked. The transmitted light was then guided to the spectrometer. Before entering the detection channel, the light emitted from the input fiber was collimated via one 3D microlens. The other 3D microlens was used to couple the light leaving the detection channel to the output fiber. These two microlenses were accurately positioned in the mold fabrication step to ensure alignment with the optical path. A detailed description of the performance of such microlenses can be found elsewhere. ${ }^{11}$

2.2. Operation of the Optical Switches. Two transparent liquids, ultrapure water and Novec HFE7500 oil (3M, St. Paul, USA) loaded with $1 \%(\mathrm{w} / \mathrm{w})$ fluorosurfactant (RAN Biotechnologies, Beverly, USA), were selected as "lighttransmitting" fluids in our experiments. A black dye solution of reactive black 5 (Sigma, St. Louis, USA) was selected as the "light-blocking" fluid. Various concentrations of dye solution $(0-20 \%, \mathrm{w} / \mathrm{w})$ were prepared to assess light blocking performance. The two combinations of ultrapure water/dye solution and oil/dye solution were evaluated for the optical switching performance. The working mechanism of the pneumatic microvalves is described in Figure S3. Briefly, when the pressure in the valve control layer (the control pressure) is significantly higher than the pressure driving the fluid (the driving pressure), the valve is closed. When the control pressure is minimized, the valve turns open. In the current study, a driving pressure of $0.04 \mathrm{MPa}$ and a control pressure of $0.14 \mathrm{MPa}$ were used in all experiments.

2.3. Analytical Methods. Nitrite and ammonium concentrations are two key parameters used in the assessment of food and water quality, with colorimetric detection being one of the most popular methods for species quantification. ${ }^{43-45}$ Accordingly, we validated the performance of OSEMC for colorimetric analysis through measurement of nitrite and ammonium samples. Specifically, nitrite was quantified according to the standard Griess reagent method. ${ }^{46}$ Ammonium content was analyzed according to the salicylate method. ${ }^{47,48}$ After being incubated in a water bath at $37^{\circ} \mathrm{C}$, samples containing nitrite and ammonium were loaded using the peristaltic pump at a flow rate of $8.5 \mu \mathrm{L} \cdot \mathrm{min}^{-1}$. All samples were measured in parallel in the four detection channels of the OSEMC. As a control, additional "parallel measurements" were performed in a 96-well plate using an Infinite $200 \mathrm{PRO}$ reader (Tecan, Männedorf, Switzerland).

Activated charcoal is commonly used for the color removal in the treatment and purification of water and wastewater, ${ }^{49-51}$ with the laboratory characterization of the adsorption kinetics of chromophores on activated charcoal being necessary to optimize relevant parameters, e.g., dose, retention time for the pilot, or large-scale operation. ${ }^{52,53}$ That said, conventional manual batch-sampling is challenging to acquire real-time data and makes this process laborious. Accordingly, we used the OSEMC system to characterize the adsorption kinetics of 100mesh activated charcoal (Sigma, St. Louis, USA) in a real-time fashion, using tartrazine with a $\lambda_{\max }$ at $428 \mathrm{~nm}$ (Sigma, St. Louis, USA) as a model target. Kinetic measurements on two doses of activated charcoal (480 and $1190 \mathrm{ppm}$ in the adsorption system) were performed. The adsorption experiment was conducted in a beaker where tartrazine solution and the activated charcoal were continuously stirred by a rotator at a speed of $50 \mathrm{rpm}$ at $24 \pm 0.5{ }^{\circ} \mathrm{C}$. Simultaneously, the mixed sample was delivered to the four detection channels at a flow 

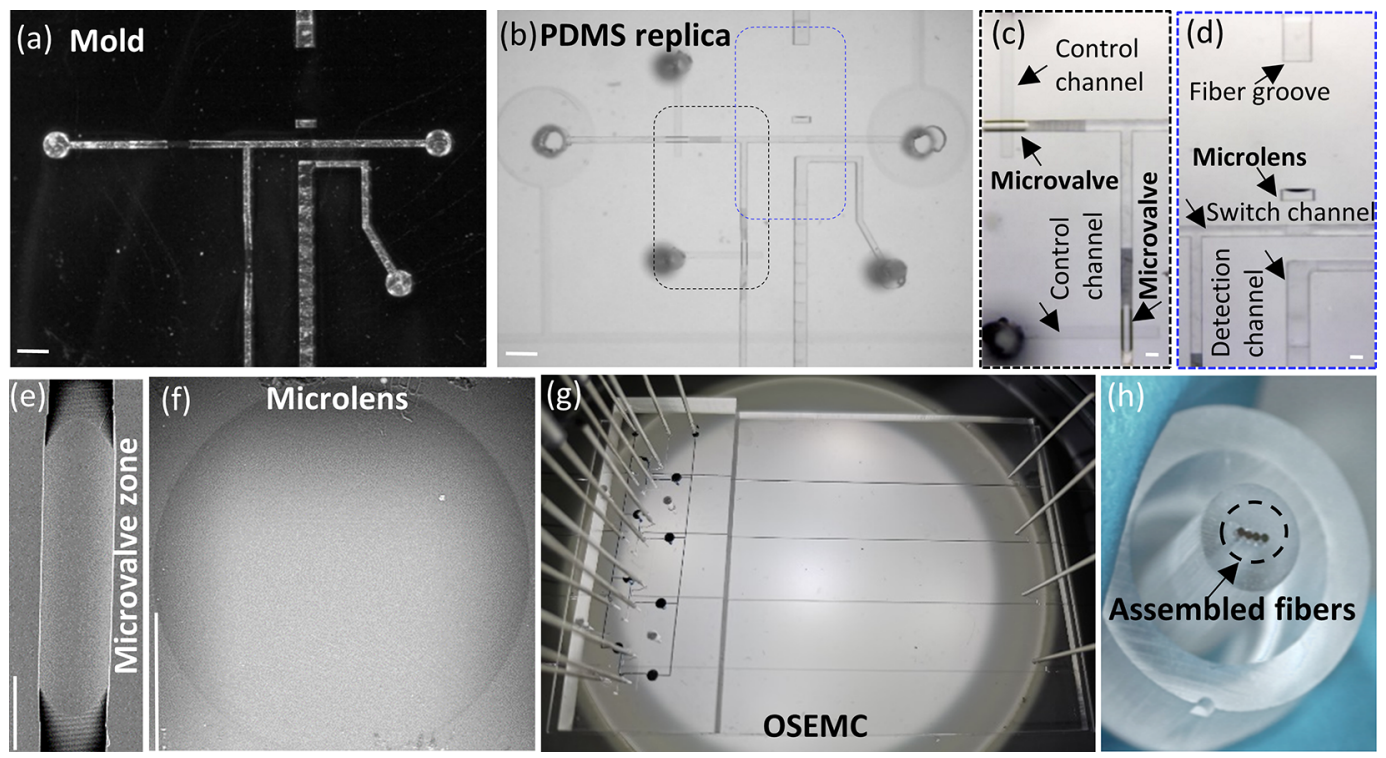

Figure 2. Fabrication of the OSEMC and optical fiber assembly. (a) The fabricated mold after the uncured photoresist has been removed. (b) The PDMS replica corresponding to the mold structure shown in part a. ( $\mathrm{c}$ and $\mathrm{d}$ ) Magnified regions in part b marked by black and blue dashed boxes highlight microvalve and optical structures. (e) SEM image of the microvalve zone. (f) SEM image of a microlens. (g) Overview of the actual OSEMC showing tubing connections. (h) Assembled optical fibers within a homemade adapter. Scale bars in parts a and b, $400 \mu \mathrm{m}$; scale bars in other images, $100 \mu \mathrm{m}$.

rate of $17.0 \mu \mathrm{L} \cdot \mathrm{min}^{-1}$ for parallel extraction of kinetic data. A $10 \mu \mathrm{L}$ pipet tip containing an embedded filter was incorporated at the entrance of the peristaltic tubing for the separation and removal of the activated charcoal. The exit of peristaltic tubing was connected to the sample inlet of OSEMC.

HRP conjugated antibody is widely used in pathogen detection using enzyme-linked immunoassays. ${ }^{54,55}$ HRP acts as a catalyst in accelerating the reaction between hydrogen peroxide and 3,3',5,5'-tetramethylbenzidine (TMB) to yield a blue color $\left(\lambda_{\max }\right.$ at $\left.650 \mathrm{~nm}\right)$, which can be directly quantified by colorimetric analysis. Specifically, ab205718 HRP conjugated antibody (Abcam, Cambridge, U.K.) samples at various concentrations $(0.02,0.05,0.10$, and $0.25 \mathrm{ppb})$ were mixed with a TMB substrate solution (Thermo Fisher Scientific, Waltham, USA) at a volume ratio of 4:1. After mixing, the samples at four different concentrations were immediately loaded into the four detection channels, respectively, at a flow rate of $17.0 \mu \mathrm{L} \cdot \mathrm{min}^{-1}$ for the simultaneous characterization of reaction kinetics at $24 \pm 0.5$ ${ }^{\circ} \mathrm{C}$. All measurements were repeated three times.

\section{RESULTS AND DISCUSSION}

3.1. Chip Fabrication and Fiber Assembly. Figure 2a illustrates the printed mold structures of the switch and detection layer after the uncured photoresist has been removed. Figure $2 \mathrm{~b}$ presents the corresponding PDMS layer manufactured via mold replication. The sections delineated by black and blue dashed boxes are enlarged and presented in Figure $2 \mathrm{c}$ and $\mathrm{d}$, clearly showing the microvalves and optical structures. Due to the use of high-resolution printing parameters, microvalves and microlenses were fabricated with excellent surface quality, as shown in the SEM images in Figure $2 \mathrm{e}$ and $\mathrm{f}$. It is evident that, when compared to conventional UV lithography, two-photon stereolithography allows for the direct fabrication of complex microstructures with a variety of dimensions and surface curvatures. Figure $2 \mathrm{~g}$ shows an assembled OSEMC chip containing four detection channels. Due to the central supply of the switch fluids, tubing connections are dramatically simplified. Additionally, the multiple bare optical fibers are assembled in a homemade adapter based on the slit dimensions of the spectrometer, as shown in Figure $2 \mathrm{~h}$. Since the assembled optical fibers operate in sequence via optical switches, four-channel detection is realized using a single light source and spectrometer.

3.2. "Light-Transmitting" and "Light-Blocking" Fluids as Optical Switches. In the current study, transparent and opaque fluid volumes were used to enable and block light transmission, respectively, and to form an optical switch. Specifically, we compared the switching performance of two fluid systems: oil/dye solution and ultrapure water/dye solution. For the oil/dye solution system, it was found that the dye fluid could not be completely displaced when switching from the "light-blocking" fluid (dye solution) to the "light-transmitting" fluid (oil). Split fragments of dye fluid adsorbed on the channel walls, as can be seen in Figure 3a1, which led to unstable optical switching. This issue is most likely related to interfacial instability. In the current scenario, the "light-blocking" fluid in the form of a liquid plug wets a large area of the PDMS channel surface and leads to high viscous force at the walls. Additionally, due to the high flow rate during fluid switching, inertial forces exerted by the incoming oil are able to overcome the surface tension at the oil-dye fluid interface. As previously observed by Kazoe and co-workers for gas-liquid flows, ${ }^{56}$ both factors likely contribute to splitting. Conversely, in the ultrapure water/ dye solution system, the "light-transmitting" fluid (ultrapure water) and the "light-blocking" fluid (dye solution) could be cleanly manipulated, as shown in Figure 3a2. Additionally, it is important to note that, when the optical switch is in the "onstate", diffusion of residual dye molecules does not affect operation, since the valve controlling the "light-transmitting" fluid is kept open during the signal readout process. When the optical switch is in the "off-state", the limited residual volume 

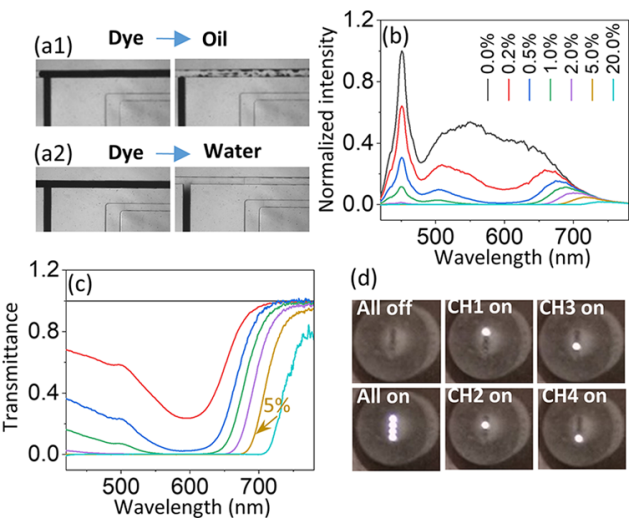

(d)

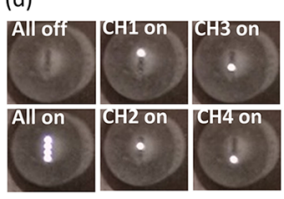

Figure 3. Fluid manipulation based on optical switching. (a) Performance of two pairs of "light-blocking" and "light-transmitting" fluids: The upper panel (a1) shows the oil/dye solution pair and the lower panel (a2) the ultrapure water/dye solution pair. (b) The dependence of light attenuation through a switch channel on the dye concentration $(0-20 \%, w / w)$. (c) The variation of light transmittance with dye concentration. (d) Switch-on or switch-off states. Images correspond to all output fibers switched off, all fibers switched on, and only a single fiber of the four switched on. The state of "on" and "off" here corresponds to the "light-transmitting" using ultrapure water and "light-blocking" using a 5\% dye solution, respectively. $\mathrm{CH}$ abbreviates the detection channel.

of ultrapure water has a negligible influence on the "lightblocking" fluid containing a high concentration of dye. As depicted in Figure $3 b$, as the dye concentration is increased, the extinction coefficient of the "light-blocking" fluid is augmented and the blocking range of the spectrum becomes broader. Correspondingly, the change of light transmittance as a function of wavelength is described in Figure 3c. In the current study, light is defined as being "blocked" if the transmittance was lower than $0.5 \%$. Accordingly, as the dye concentration of the "light-blocking" fluid is increased from 0 to $5 \%$, light between 420 and $670 \mathrm{~nm}$ could be blocked. At a concentration of $20.0 \%$, the blocking range could be further extended to $710 \mathrm{~nm}$. That said, since the primary absorption peaks for all species in the current studies are located between 428 and $650 \mathrm{~nm}$, a $5 \%$ dye solution was deemed sufficient. Indeed, light exiting the output fiber could be tuned into the states "all-off", "all-on", and "single-on", as presented in Figure $3 \mathrm{~d}$.

3.3. Performance of the Optical Switches. Response time, switching frequency, and stability are the key performance parameters of the optical switches. Intensity variations over five cycles are shown in Figure 4a, with data indicating excellent response time consistency between the four channels. On average, the response time for both "switch-on" and "switch-off" processes (covering two to three data points) is approximately $200 \mathrm{~ms}$, which is considerably shorter than those reported in several prior studies. For example, Cao et al. and Dietvorst et al. developed microbubble-based and microfluid-based optical routers, achieving response times of 1.8 and $3.3 \mathrm{~s}$, respectively. ${ }^{21,57}$ Importantly, the short response time accessible in our system is suitable for multichannel absorbance measurements and the extraction of kinetic data with a time resolution on the second scale. It should be noted, although faster optical switching has been achieved using a TIR-based droplet approach, ${ }^{19}$ it is relatively difficult to control droplet position for the controllable multichannel applications. In contrast, by virtue of the use of pneumatic microvalves, each of the "light-transmitting" and "lightblocking" fluid entities in the current system can be independently manipulated and positioned, allowing program-
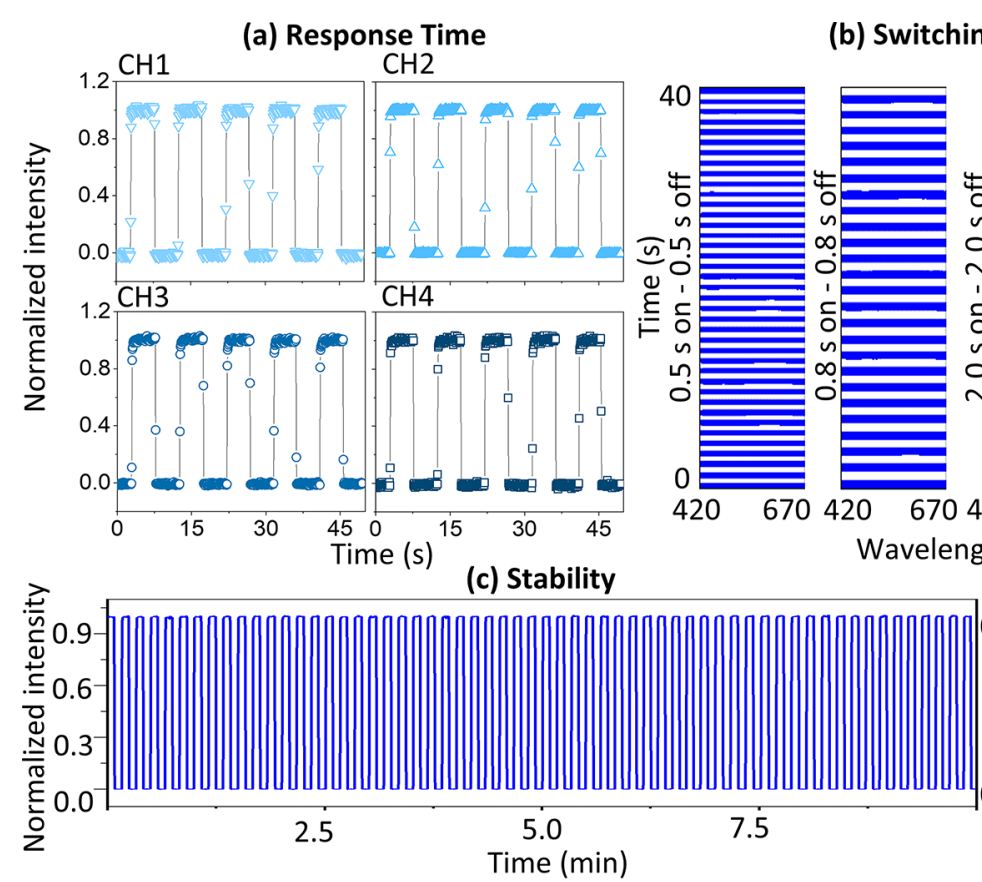

(b) Switching Frequency

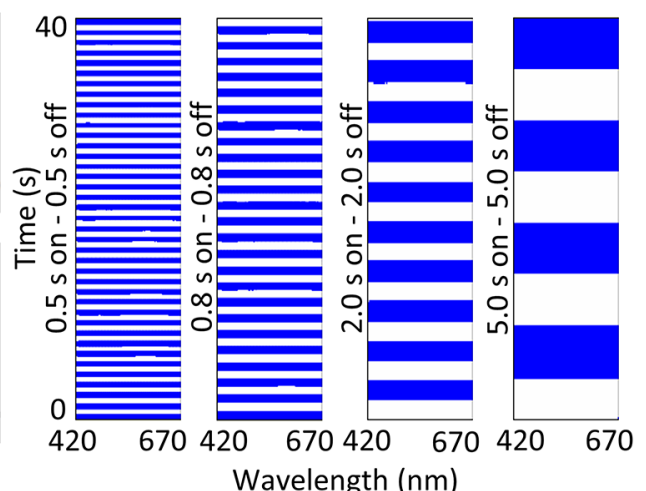

(d)

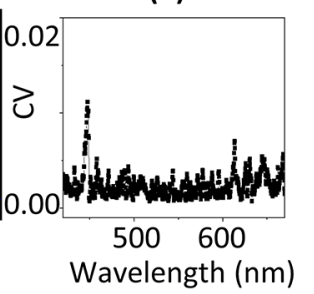

Figure 4. Optical switch performance. (a) Response time of the optical switches for each of the four detection channels. (b) Switching frequency tuning. From left to right: $0.5 \mathrm{~s}$ on $-0.5 \mathrm{~s}$ off $(1.0 \mathrm{~Hz}), 0.8 \mathrm{~s}$ on- $0.8 \mathrm{~s}$ off $(0.625 \mathrm{~Hz}), 2.0 \mathrm{~s}$ on $-2.0 \mathrm{~s}$ off $(0.25 \mathrm{~Hz}), 5.0 \mathrm{~s}$ on $-5.0 \mathrm{~s}$ off $(0.1 \mathrm{~Hz})$. (c) Stability of the optical switch over multiple cycles of operation. (d) The CV profile within the blocking range. The light intensity in parts a and c was measured at $550 \mathrm{~nm}$ and normalized with the average value corresponding to the "switch-on" state. All four detection channels were filled with ultrapure water. 
mable operation in multichannel formats. Figure $4 \mathrm{~b}$ presents the tunability of the optical switch in regard to switching frequency between 0.1 and $1.0 \mathrm{~Hz}$. Figure $4 \mathrm{c}$ shows the intensity responses at $550 \mathrm{~nm}$ over 60 cycles and $10 \mathrm{~min}$ (operated at $0.1 \mathrm{~Hz}$ ). Significantly, the mean coefficient variation $(\mathrm{CV})$ of this intensity between 420 and $670 \mathrm{~nm}$ was $0.24 \pm 0.13 \%$, confirming the excellent stability of the optical switch. For the operations at frequencies of $0.25,0.625$, and 1.0 $\mathrm{Hz}$, the CV within 120 cycles (shown in Figure S4) was in the range of $0.64 \pm 0.24 \%$. The process of optical switching, changes of output fiber states, and the corresponding spectral changes can be seen in video S2, video S3, and video S4, respectively.

3.4. Reproducibility and Sensitivity. The reproducibility of switching between the four detection channels is of importance when assessing the performance of the OSEMC platform in multichannel applications. To assess this issue, nitrite and ammonium samples were analyzed. Parts a and b of Figure 5 report the absorption spectra obtained from incubated
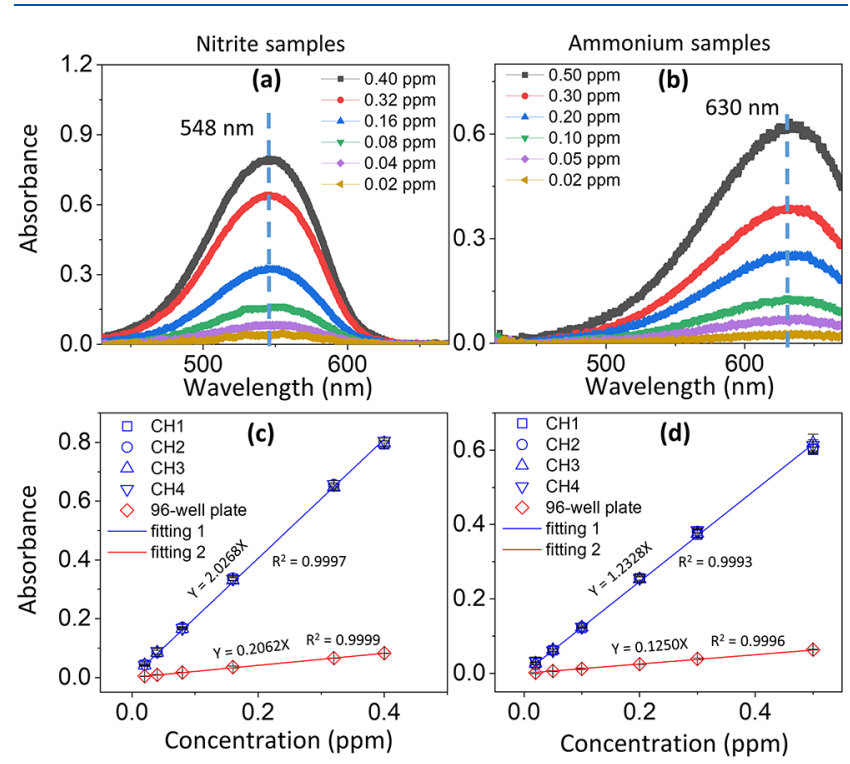

Figure 5. Assessment of multichannel absorbance measurements and analytical sensitivity for an OSEMC-based assay and a 96-well platebased assay. (a, b) Absorption spectra for nitrite $\left(\lambda_{\max }=548 \mathrm{~nm}\right)$ and ammonium $\left(\lambda_{\max }=630 \mathrm{~nm}\right)$ samples, respectively. $(c, d)$ Variation of absorbance as a function of concentration for the OSEMC-based assay and 96-well plate-based assay, respectively. The lines of fitting 1 and fitting 2 in parts $\mathrm{c}$ and $\mathrm{d}$ represent the linear regression analysis of the results from the four detection channels of OSEMC and from the 96-well plate, respectively. The $\mathrm{LoD}$ values were derived from the regression equation based on three times of standard deviation of absorbance for blank samples. All absorbance data represented the net value after subtracting the level of blank samples. Error bars represent the standard deviation of results from three repeat measurements. A switching frequency of $0.1 \mathrm{~Hz}$ was used for all experiments in this section.

nitrite and ammonium samples at various concentrations, respectively. The measurement results of nitrite presented in Figure 5c from the four detection channels show good consistency, since all data follow faithfully a global linear equation with the $R$-square value higher than 0.999. A similarly satisfactory global linear relationship is established as well for the measurement results of ammonium presented in Figure 5d. The average coefficients of absorbance variations among the four detection channels were $1.4 \pm 0.8 \%$ and $1.5 \pm 1.4 \%$ for nitrite and ammonium detection, respectively, confirming the suitability of the OSEMC platform for parallel measurements. Additionally, comparison between the calculated concentrations according to the regression equations and the known concentrations of the prepared samples yielded deviations of $3.4 \pm 2.2 \%$ and $3.2 \pm 4.3 \%$ for nitrite and ammonium, respectively, demonstrating good measurement accuracy. Noticeably, the sensitivity of the OSEMC-based assay $(3 \mu \mathrm{L}$ volume detection channel with $60 \mathrm{~mm}$ optical pathway) was approximately 10 times higher than that of the 96-well-platebased assay $(200 \mu \mathrm{L}$ detection well with $6.2 \mathrm{~mm}$ optical pathway), according to the slope values of the linear regression equations for all of the sample measurements. Correspondingly, the limit of detection (LoD) values for nitrite and ammonium detection based on the OSEMC assay were determined to be 0.9 and $3.1 \mathrm{ppb}$, respectively. These represent a significant improvement compared to those (5.8 and $9.6 \mathrm{ppb}$ ) originating from the 96-well plate assay.

3.5. Real-Time Characterization of Adsorption and Reaction Kinetics. Multichannel colorimetric analysis using the OSEMC platform has obvious utility in the kinetic measurements. The advantage of multichannel chips over single-channel ones is especially pronounced for kinetic measurements which usually require longer time than analysis of a single state of the sample. Additionally, it is convenient to measure the real-time results of the flowing samples within microfluidic chips. In this study, we investigated the performance of OSEMC for parallel measurements of the adsorption kinetics of tartrazine on activated charcoal. Kinetic measurements described in section 2.3 were made using two different concentrations of activated charcoal (480 and $1190 \mathrm{ppm}$ ). As can be seen in Figure 6a, data from the four detection channels were highly reproducible at both concentrations of activated charcoal, with the tartrazine removal rate reaching $90 \%$ after 10 min of adsorption by $1190 \mathrm{ppm}$ activated charcoal. In contrast, the adsorption of tartrazine was much slower when using a lower concentration of activated charcoal. In addition to parallel measurement of adsorption kinetics, the solutions with HRP conjugated antibody concentrations of $0.02,0.05,0.10$, and $0.25 \mathrm{ppb}$ were respectively loaded to the four channels, which enabled characterization of the reaction kinetics at different concentrations simultaneously (Figure 6b). As can be seen, reaction rates were enhanced by 14 times when the HRP conjugated antibody concentration increased from 0.02 to 0.25 ppb. Noticeably, had a single channel chip been used for the same aforementioned kinetics characterization, the needed time would have been 4 times as long.

\section{CONCLUSIONS}

Through the use of two-photon stereolithography, we have successfully developed and characterized an optical-switchenabled microfluidic chip for sensitive multichannel colorimetric analysis. The integrated optical switches operate in a user-defined sequence when manipulating "light-transmitting" and "light-blocking" fluids via programmable pneumatic microvalves. Fast response time (approximately $200 \mathrm{~ms}$ ), tunable switching frequencies $(0.1-1.0 \mathrm{~Hz}$ tested in this study), and excellent switching stability (mean CV: $0.54 \pm$ $0.28 \%$ ) ensure that the OSEMC platform is suitable for flowbased absorbance measurements and the extraction of adsorption and reaction kinetics. The excellent sensitivity and reproducibility between the four detection channels used 


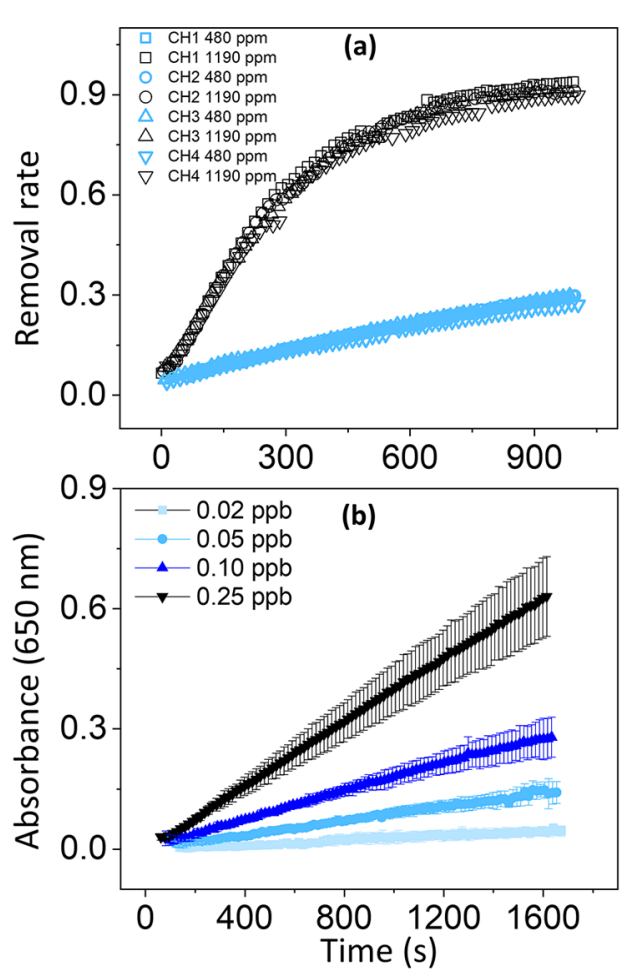

Figure 6. Characterization of adsorption and reaction kinetics using the OSEMC system. (a) The adsorption kinetics of tartrazine (4.76 ppm solution, used as a model target) on activated charcoal using different concentrations (480 and $1190 \mathrm{ppm}$ ). In each test, the same sample was simultaneously loaded into the four detection channels. (b) The reaction kinetics of TMB colorization triggered by HRP conjugated antibody. Samples catalyzed by various concentrations of HRP conjugated antibody $(0.02-0.25 \mathrm{ppb})$ were simultaneously analyzed in the four channels. The error bars represent the standard deviation of absorbance in three repeated measurements. A switching frequency of $0.25 \mathrm{~Hz}$ was used for all experiments in this section.

in the current system allow for parallel and real-time colorimetric measurements with an improved sensitivity and enhanced throughput. Besides optical switches, light-absorption-based optofluidic configurations can also play a role in light attenuators or filters via tuning the light absorption efficiencies or colors of the modulating liquids.

\section{ASSOCIATED CONTENT}

\section{SI Supporting Information}

The Supporting Information is available free of charge at https://pubs.acs.org/doi/10.1021/acs.analchem.1c00674.

Video S1 showing the real-time printing of key structures via two-photon stereolithography (MP4)

Video S2 showing the movement of light-transmitting and light-blocking fluids at 38 various optical switching frequencies (MP4)

Video S3 showing the induced changes of light emitted from the output fiber (MP4)

Video S4 showing real-time spectral changes as a result of the optical switching at 46 various frequencies (MP4) Figure S1 showing microfluidic chip molds on silicon wafers, Figure S2 showing key structures printed in the photoresist prior to development, Figure S3 showing a schematic representation of the working states of the pneumatic valve, and Figure S4 showing the switching stability over 120 cycles for three switching frequencies (PDF)

\section{AUTHOR INFORMATION}

\section{Corresponding Author}

Jing Wang - Institute of Environmental Engineering, ETH Zürich, Zürich 8093, Switzerland; Laboratory for Advanced Analytical Technologies, Empa, Swiss Federal Laboratories for Materials Science and Technology, Dübendorf 8600, Switzerland; 이이. orcid.org/0000-0003-2078-137X; Email: jing.wang@ifu.baug.ethz.ch

\section{Authors}

Jiukai Tang - Institute of Environmental Engineering, ETH Zürich, Zürich 8093, Switzerland; Laboratory for Advanced Analytical Technologies, Empa, Swiss Federal Laboratories for Materials Science and Technology, Dübendorf 8600, Switzerland

Xiaobao Cao - Institute of Chemical and Bioengineering, ETH Zürich, Zürich 8093, Switzerland; 이이.orid.org/0000-00032211-2823

Guangyu Qiu - Institute of Environmental Engineering, ETH Zürich, Zürich 8093, Switzerland; Laboratory for Advanced Analytical Technologies, Empa, Swiss Federal Laboratories for Materials Science and Technology, Dübendorf 8600, Switzerland

Andrew deMello - Institute of Chemical and Bioengineering, ETH Zürich, Zürich 8093, Switzerland; (1) orcid.org/00000003-1943-1356

Complete contact information is available at:

https://pubs.acs.org/10.1021/acs.analchem.1c00674

\section{Author Contributions}

J.T. conceived of the idea and implemented all experiments. J.T., A.D., and J.W. wrote the manuscript. X.C. assisted in establishing the programmable pneumatic valve system and provided guidance in the fabrication of microfluidic chips. G.Q. assisted in establishing the optical system and made comments on the manuscript. A.D. provided the facilities for the fabrication of microfluidic devices. J.W. supervised the work.

\section{Notes}

The authors declare no competing financial interest.

\section{ACKNOWLEDGMENTS}

We thank Mr. Yingchao Meng and Mr. Yanming Xia for the assistance with UV lithography and printing of the fiber adapter. We also thank Dr. Huan Liu for help with the absorbance measurements of nitrite and ammonium samples.

\section{REFERENCES}

(1) Sieben, V. J.; Floquet, C. F.; Ogilvie, I. R.; Mowlem, M. C.; Morgan, H. Anal. Methods 2010, 2 (5), 484-491.

(2) Ziyaina, M.; Rasco, B.; Coffey, T.; Ünlü, G.; Sablani, S. S. Food Control 2019, 100, 220-226.

(3) Weston, M.; Kuchel, R. P.; Ciftci, M.; Boyer, C.; Chandrawati, R. J. Colloid Interface Sci. 2020, 572, 31-38.

(4) Liu, B.; Zhuang, J.; Wei, G. Environ. Sci.: Nano 2020, 7 (8), 2195-2213.

(5) Zhu, J. M.; Shi, Y.; Zhu, X. Q.; Yang, Y.; Jiang, F. H.; Sun, C. J.; Zhao, W. H.; Han, X. T. Lab Chip 2017, 17 (23), 4025-4030.

(6) Shi, Y.; Liu, H. L.; Zhu, X. Q.; Zhu, J. M.; Zuo, Y. F.; Yang, Y.; Jiang, F. H.; Sun, C. J.; Zhao, W. H.; Han, X. T. Lab Chip 2018, 18 (19), 2994-3002. 
(7) Zheng, L.; Cai, G.; Wang, S.; Liao, M.; Li, Y.; Lin, J. Biosens. Bioelectron. 2019, 124-125, 143-149.

(8) Oh, S. J.; Park, B. H.; Choi, G.; Seo, J. H.; Jung, J. H.; Choi, J. S.; Kim, D. H.; Seo, T. S. Lab Chip 2016, 16 (10), 1917-1926.

(9) Choi, J.; Baek, J.; Kweon, D.; Ko, K. S.; Yoon, H. J. Microbiol. 2020, 58, 415-421.

(10) Lafhal, K.; Sabir, E.-s.; Cheggour, M.; Mouad, F.; Hammoud, M.; Lalaoui, A.; Ezoubeiri, A.; Baki, S.; Chabaa, L.; Maoulainine, F. M. R.; Rada, N.; Bouskraoui, M.; Ait Saab, I.; Bourrahouat, A.; Fdil, N. Carbohydr. Res. 2020, 498, 108179.

(11) Tang, J.; Qiu, G.; Cao, X.; Yue, Y.; Zhang, X.; Schmitt, J.; Wang, J. Lab Chip 2020, 20 (13), 2334-2342.

(12) Vila-Planas, J.; Fernández-Rosas, E.; Ibarlucea, B.; Demming, S.; Nogués, C.; Plaza, J. A.; Domínguez, C.; Büttgenbach, S.; Llobera, A. Nat. Protoc. 2011, 6 (10), 1642-1655.

(13) Zhu, J.; Han, G.; Hu, X.; Zuo, Y.; Chen, L.; Wang, F.; Yang, Y.; Jiang, F.; Sun, C.; Zhao, W.; Han, X. ACS Sensors 2020, 5 (5), 13811388.

(14) Mei, H.; Pan, J.; Zhang, Z.; Zhang, L.; Tong, L. ACS Sensors 2019, 4 (9), 2267-2271.

(15) Lynch, K. B.; Yang, Y.; Ren, J.; Liu, S. Talanta 2018, 181, 416421.

(16) Zhang, L.; Pan, J.; Zhang, Z.; Wu, H.; Yao, N.; Cai, D.; Xu, Y.; Zhang, J.; Sun, G.; Wang, L.; Geng, W.; Jin, W.; Fang, W.; Di, D.; Tong, L. Opto-Electronic Advances 2020, 3 (3), 19002201-19002207.

(17) Pan, J.; Jiang, C.; Zhang, Z.; Zhang, L.; Wang, X.; Tong, L. Advanced Materials Technologies 2020, 5 (6), 2000079.

(18) Horsley, D. A.; Davis, W. O.; Hogan, K. J.; Hart, M. R.; Ying, E. C.; Chaparala, M.; Behrang, B.; Daneman, M. J.; Meng-Hsiung, K. J. Microelectromech. Syst. 2005, 14 (2), 274-284.

(19) Oraie, M.; Latifi, H.; Qazvini, H.; Nikbakht, H.; Ghaderzadeh, A. IEEE Photonics Technol. Lett. 2017, 29 (16), 1383-1386.

(20) Wan, J.; Miao, J.; Xu, P.; Zhang, S.; Duan, Y. Opt. Commun. 2020, 454, 124378.

(21) Dietvorst, J.; Goyvaerts, J.; Ackermann, T. N.; Alvarez, E.; Muñoz-Berbel, X.; Llobera, A. Lab Chip 2019, 19 (12), 2081-2088.

(22) Xu, P.; Wan, J.; Zhang, S.; Duan, Y.; Chen, B.; Zhang, S. Appl. Opt. 2019, 58 (17), 4637-4641.

(23) Liu, C.; Li, L.; Wang, Q.-H. J. Appl. Phys. 2013, 113 (19), 193106.

(24) Ren, H.; Wu, S.-T. Opt. Lett. 2010, 35 (22), 3826-3828.

(25) Zohrabi, M.; Lim, W. Y.; Bright, V. M.; Gopinath, J. T. Opt. Express 2020, 28 (5), 5991-6001.

(26) Mibus, M.; Jensen, C.; Hu, X.; Knospe, C.; Reed, M.; Zangari, G. J. Appl. Phys. 2013, 114 (1), No. 014901.

(27) Shen, H.-H.; Chung, L.-Y.; Yao, D.-J. Biomicrofluidics 2015, 9 (2), No. 022403.

(28) Kim, H. S.; Weiss, T. L.; Thapa, H. R.; Devarenne, T. P.; Han, A. Lab Chip 2014, 14 (8), 1415-1425.

(29) Unger, M. A.; Chou, H.-P.; Thorsen, T.; Scherer, A.; Quake, S. R. Science 2000, 288 (5463), 113-116.

(30) Whitesides, G. M. Nature 2006, 442 (7101), 368-373.

(31) Kim, J.; Stockton, A. M.; Jensen, E. C.; Mathies, R. A. Lab Chip 2016, 16 (5), 812-819.

(32) Rho, H. S.; Yang, Y.; Terstappen, L. W. M. M.; Gardeniers, H.; Le Gac, S.; Habibović, P. J. Ind. Eng. Chem. 2020, 91, 231-239.

(33) Zeng, S.; Li, B.; Su, X. o.; Qin, J.; Lin, B. Lab Chip 2009, 9 (10), $1340-1343$.

(34) Sun, Y.; Cai, B.; Wei, X.; Wang, Z.; Rao, L.; Meng, Q.-F.; Liao, Q.; Liu, W.; Guo, S.; Zhao, X. Electrophoresis 2019, 40 (6), 961-968.

(35) Fu, A. Y.; Chou, H.-P.; Spence, C.; Arnold, F. H.; Quake, S. R. Anal. Chem. 2002, 74 (11), 2451-2457.

(36) Hong, J. W.; Studer, V.; Hang, G.; Anderson, W. F.; Quake, S. R. Nat. Biotechnol. 2004, 22 (4), 435-439.

(37) Park, W.; Han, S.; Kwon, S. Lab Chip 2010, 10 (20), 28142817.

(38) Aćimović, S. S.; Ortega, M. A.; Sanz, V.; Berthelot, J.; GarciaCordero, J. L.; Renger, J.; Maerkl, S. J.; Kreuzer, M. P.; Quidant, R. Nano Lett. 2014, 14 (5), 2636-2641.
(39) Guo, F.; Lapsley, M. I.; Nawaz, A. A.; Zhao, Y.; Lin, S.-C. S.; Chen, Y.; Yang, S.; Zhao, X.-Z.; Huang, T. J. Anal. Chem. 2012, 84 (24), 10745-10749.

(40) Shi, J.; Stratton, Z.; Lin, S.-C. S.; Huang, H.; Huang, T. J. Microfluid. Nanofluid. 2010, 9 (2), 313-318.

(41) Cao, X.; Du, Y.; Küffner, A.; Van Wyk, J.; Arosio, P.; Wang, J.; Fischer, P.; Stavrakis, S.; deMello, A. Small 2020, 16 (20), 1907534.

(42) Gissibl, T.; Thiele, S.; Herkommer, A.; Giessen, H. Nat. Photonics 2016, 10 (8), 554-560.

(43) Nam, J.; Jung, I.-B.; Kim, B.; Lee, S.-M.; Kim, S.-E.; Lee, K.-N.; Shin, D.-S. Sens. Actuators, B 2018, 270, 112-118.

(44) Trofimchuk, E.; Hu, Y.; Nilghaz, A.; Hua, M. Z.; Sun, S.; Lu, X. Food Chem. 2020, 316, 126396.

(45) Huang, J.; Kankanamge, N. R.; Chow, C.; Welsh, D. T.; Li, T.; Teasdale, P. R. J. Environ. Sci. 2018, 63, 174-197.

(46) Griess reagent kit for nitrite determination G-7921. Molecular Probes: 2003.

(47) Hu, J.; Zhao, Y.; Yang, W.; Wang, J.; Liu, H.; Zheng, P.; Hu, B. FEMS Microbiol. Ecol. 2020, 96 (10), fiaa179.

(48) Bower, C. E.; Holm-Hansen, T. Can. J. Fish. Aquat. Sci. 1980, 37 (5), 794-798.

(49) Patel, S. K.; Patel, S. G.; Patel, G. V. Proc. Natl. Acad. Sci., India, Sect. A 2020, 90, 579.

(50) Bernal, M.; Ruiz, M. O.; Geanta, R. M.; Benito, J. M.; Escudero, I. Chem. Eng. J. 2016, 283, 313-322.

(51) Collivignarelli, M. C.; Abbà, A.; Carnevale Miino, M.; Damiani, S. J. Environ. Manage. 2019, 236, 727-745.

(52) Özbay, I.; Özdemir, U.; Özbay, B.; Veli, S. Desalin. Water Treat. 2013, 51 (31-33), 6091-6100.

(53) Andersson, K. I.; Eriksson, M.; Norgren, M. Ind. Eng. Chem. Res. 2011, 50 (13), 7733-7739.

(54) Ahmed, A.; Rushworth, J. V.; Wright, J. D.; Millner, P. A. Anal. Chem. 2013, 85 (24), 12118-12125.

(55) Altintas, Z.; Akgun, M.; Kokturk, G.; Uludag, Y. Biosens. Bioelectron. 2018, 100, 541-548.

(56) Kazoe, Y.; Matsuno, T.; Yamashiro, I.; Mawatari, K.; Kitamori, T. Micromachines 2018, 9 (9), 423.

(57) Cao, K.; Liu, Y.; Lang, C.; Xu, Q.; Zhao, Y. Opt. Lett. 2020, 45 (14), 3901-3904. 